\title{
AKR1D1*36 C>T (rs1872930) allelic variant is associated with variability of the CYP2C9 genotype predicted pharmacokinetics of ibuprofen enantiomers - a pilot study in healthy volunteers
}

\author{
ALEKSANDRA KAPEDANOVSKA NESTOROVSKA ${ }^{1}$ \\ KRUME JAKJOVSKI ${ }^{2}$ \\ ZORICA NAUMOVSKA ${ }^{1}$ \\ ZORAN STERJEV ${ }^{1}$ \\ NADICA MATEVSKA GESKOVSKA ${ }^{1}$ \\ KRISTINA MLADENOVSKA ${ }^{1}$ \\ LJUBICA SUTURKOVA ${ }^{1}$ \\ ALEKSANDAR DIMOVSKI ${ }^{1,3}$ \\ ${ }^{1}$ Center for Biomolecular and Pharmaceutical \\ Analysis, Faculty of Pharmacy, University \\ Ss Cyril and Methodius, Skopje 1000 \\ Republic of Macedonia \\ ${ }^{2}$ Department of Preclinical and Clinical \\ Pharmacology and Toxicology \\ Faculty of Medicine, University Ss Cyril \\ and Methodius, Skopje 1000 \\ Republic of Macedonia \\ ${ }^{3}$ Research Center for Genetic Engineering \\ and Biotechnology "Georgi D. Efremov" \\ Macedonian Academy of Sciences and Arts \\ 1000 Skopje, Republic of Macedonia
}

Accepted February 18, 2019

Published online March 22, 2019
The relative contribution of CYP2C9 allelic variants to the pharmacokinetics (PK) of ibuprofen (IBP) enantiomers has been studied extensively, but the potential clinical benefit of pharmacogenetically guided IBP treatment is not evident yet. The role of AKR1D1*36C $>\mathrm{T}$ (rs 1872930) allelic variant in interindividual variability of CYP450 mediated drug metabolism was recently elucidated. A total of 27 healthy male subjects, volunteers in IBP single-dose two-way cross-over bioequivalence studies were genotyped for CYP2C9*2, CYP2C9*3 and AKR1D1*36 polymorphisms. The correlation between CYP2C9 and AKR1D1 genetic profile and the PK parameters for $S-(+)$ and $R-(-)$-IBP was evaluated. Remarkable changes in the PK values pointing to reduced CYP2C9 enzyme activity were detected only in the CYP2C9*2 allelic variant carriers. Statistically significant association between the AKR1D1*36 allele and the increased IBP metabolism (low $A U C_{0-\mathrm{t}}$ and ${ }_{0-\infty}$ high $C l_{\text {tot }}$ and short $t_{\max }$ values for both enantiomers) was observed in subjects carrying the CYP2C $9 * 1 / * 3$ or CYP2C $9 * 1 /{ }^{*} 1$ genotype. The clinical value of concomitant CYP2C9 and AKR1D1 genotyping has to be further verified.

Keywords: ibuprofen, enantiomers, pharmacokinetics, AKR1D1, CYP2C9, pharmacogenetics

Ibuprofen (IBP, 2-arylpropionic acid derivative [(R,S)-2-(4-(2-methylpropyl)phenyl)] propionic acid]) is probably the most widely used non-steroid anti-inflammatory drug (NSAID) today. Its short plasma half-life, wide therapeutic window, lack of development of pathologically-related metabolites and prolonged retention in specific body compartments make IBP one of the safest and probably the least toxic non-selective inhibitors of cyclooxygenase (COX-1 and COX-2) and the only NSAID approved for use in pediatric population starting from the age of 3 months $(1,2)$. In most formulations, IBP exists as a

\footnotetext{
*Correspondence, e-mail: alka@ff.ukim.edu.mk
} 
A. Kapedanovska Nestorovska et al.: AKR1D1*36 C>T (rs1872930) allelic variant is associated with variability of the CYP2C9 genotype predicted pharmacokinetics of ibuprofen enantiomers - a pilot study in healthy volunteers, Acta Pharm. 69 (2019) $399-412$.

diastereoisomeric mixture, which comprises a $S-(+)$ moiety and a $R-(-)$ enantiomer moiety. $S$-(+)-IBP is pharmacologically more active as a prostaglandin (PG) synthesis inhibitor; however, $R$-(-)-IBP has pharmacological properties relevant to the anti-inflammatory activity of IBP. In humans, $R-(-)$-IBP undergoes extensive enantiomeric conversion to the $S-(+)$ isomer and both isomers are metabolized extensively to inactive metabolites. Thus, the contribution of each of the isomers to the anti-inflammatory, analgesic and antipyretic effects of IBP underlines the interrelation between the PK/metabolism of IBP and its pharmacodynamics (PD) $(1,3,4)$. When orally administered, IBP is rapidly absorbed from the upper gastrointestinal tract, with the maximum time to reach the maximum plasma concentration $\left(t_{\max }\right)$ of approximately 1-2 $\mathrm{h}$. Depending on the release characteristics of the formulation, various plasma $S / R$ ratios have been observed, namely, higher with modified-release compared to conventional release, i.e. immediate-release formulations. The bio distribution of IBP is characterized by high plasma protein binding and low distribution volume $\left(V_{\mathrm{d}}\right)$, with significant accumulation in inflamed compartments. In subjects with preserved renal and liver functions, IBP exhibits linear kinetics when taken in up to 1200 mg (repeated) doses. Plasma concentration $\left(C_{\mathrm{p}}\right)$ and, to some extent, the area under the curve $(A U C)$ values are dose-related, but with little variation with (repeated) dosage as suggested by the kinetic constants, elimination half-life $\left(t_{1 / 2}\right) /$ elimination rate constant $\left(K_{\mathrm{el}}\right)$. The non-linear PKs occurring at high doses is due to the saturation of plasma protein binding. The plasma half-life of IBP is between 2 and $3 \mathrm{~h}$ on average, with excretion being complete after $24 \mathrm{~h}$ of the last IBP dose administration. The relatively fast rate of absorption of IBP is followed by a systematic metabolism of both enantiomers, which are extensively (99\%) metabolized to their major chiral, pharmacologically inactive 2-hydroxyl, 2-carboxyl and glucuronide metabolites via CYP450 (CYP2C8 and 2C9) mediated oxidation and UGT (UGT2B7) mediated glucuronidation. Half-lives of the S-(+)-IBP, 2-hydroxylated or carboxylated glucuronides are approximately $3.7 \mathrm{~h}$ and about $1.7 \mathrm{~h}(1,5-8)$ of $\mathrm{R}-(-)-\mathrm{IBP}$ acyl glucuronides. CYP2C9 has been identified as the most important catalyst for the formation of all oxidative metabolites of IBP enantiomers, though it is mainly involved in the hydroxylation of S-(+)-IBP, while CYP2C8 preferentially metabolizes $R-(-)-I B P(8-10)$. Polymorphisms in CYP2C8 and CYP2C9 gene ( ${ }^{*} 2$ and ${ }^{*} 3$ allelic variants) coding for the corresponding enzymes are among the identified factors with a direct impact on the PK of IBP and thus on the variability in drug response and adverse drug events (11). Major adverse events affect the gastrointestinal tract (GIT), kidneys and the coagulation system. Chronic treatment with IBP is a potential reason for the occurrence of GI bleeding, increased risk of gastric ulcers and damages and also of epistaxis, heart failure, hyperkalemia, confusion and bronchial spasms. Other adverse reactions, though very rare, were also reported, including renal adverse events such as acute renal failure, interstitial nephritis and nephritic syndrome (12-15). The incidence and intensity of ADE might vary significantly due to the CYP2C8/C9 stereospecific preference of IBP (11). However, information on interindividual variability of pharmacokinetic parameters related to the safety profile of IBP and its enantiomers is limited. Published results on the CYP2C8/C9 genetic impact on IBP metabolism are mainly inconclusive and open to doubt, mostly because of the incomplete genotype effect observed in studies with small healthy cohorts and/or heterogeneous cohorts and also because of the difficulty to distinguish the CYP2C8/C9 allelic variant effects on the IBP enantiospecific metabolism due to the high sequence homology between the two genes (16-18). Moreover, it has been found that the amount of active CYP2C9 enzyme depends on the level of gene expression, the rates of mRNA transcription and degradation, protein 
degradation, the presence of enzyme inhibitors/antagonists and post-transcriptional regulation of $\mathrm{CYP} 2 \mathrm{C}$ isoforms by microRNAs. The amount of protein of other CYP isoforms (CYP2D6, CYP3A4) might also influence CYP2C9 enzyme activity via protein-protein interactions (19). In addition, the acylation of proteins to form adducts from the reaction of acyl glucuronides of ibuprofen may be of relevance for adverse reactions in the liver analogous to those occurring with some other NSAIDs, though evidence for this is still lacking (1). Overall, even when CYP2C9 enzyme involvement is major, each COX inhibitor has to be evaluated on an individual basis, taking into account the available data related to all clearance mechanisms, direct conjugation and especially non-P450 oxidation (20).

A recently published study by Chandry et al. (21) provided a new insight, which can account for interindividual differences in CYP450 drug metabolism, drug efficacy and adverse drug events. The authors obtained experimental evidence supporting the putative role of the AKR1D1 gene as a trans-genetic regulator of the CYP network and pointed to the significant association between AKR1D1*36 C $>$ T (rs1872930) allelic variation, augmented hepatic CYP450 mRNA expression and increased activity of AKR1D1, CYP2C9 and CYP2C8 enzymes coded by the corresponding genes. AKR1D1 catalyzes the NADPH dependent reduction of bile acids and steroids but it is also involved in the reduction of either carbonyl groups or $\alpha, \beta$-unsaturated ketones on a variety of xenobiotics (dicarbonyls and polycyclic aromatic hydrocarbon trans-dihydrodiols, including a great variety of nonsteroidal aldehydes and ketones (22). Hence, it is plausible that the AKR1D1*36 C>T (rs1872930) polymorphism may contribute to the observed interindividual variability of IBP PK and the associated drug response. These recent findings are of particular importance since the most common CYP2C8/C9 cis-variations, independently and together, do not explain clearly the patient-to-patient inconsistency in the metabolism of IBP enantiomers. Despite the availability of numerous studies evaluating the genetic factors associated with interindividual variability of the IBP metabolism, there are no reported studies about the impact of AKR1D1*36C>T on the metabolism of $S-(+)$ and $R-(-)$-IBP. Having all this in mind, our pilot study aims to evaluate the separate and appraise the combined genetic impact of CYP2C9 and AKR1D1 variant alleles on the pharmacokinetics of ibuprofen enantiomers in healthy male volunteers from The Republic of Macedonia.

\section{EXPERIMENTAL}

\section{Study population}

The study sample comprised 27 healthy Caucasian male volunteers according to the medical history, ECG, vital signs, laboratory data and physical examination (non-smokers, aged 18-55 years, mean weight $81.4 \pm 10.61 \mathrm{~kg}$, BMI between 18.5 and $30.0 \mathrm{~kg} \mathrm{~m}^{-2}$ ), enrolled in four bioequivalence trials. Investigations were conducted in the Department of Preclinical and Clinical Toxicology and Pharmacology of the Faculty of Medicine (University "Ss Cyril and Methodius" in Skopje, Macedonia) after the approval of the Faculty of Medicine Ethics and Scientific Committee and authorization by the local regulatory agency. All studies were performed in accordance with the local legislation on clinical research in humans and the Helsinki Declaration (2013), following the Guidance on Good Clinical Practice (Note for Guidance on Good Clinical Practice; EMA/CHMP/ICH/135/95 London. June 2017), Guidance on the Investigation of Bioequivalence (CPMP/EWP/QWP/1401/98 
A. Kapedanovska Nestorovska et al.: AKR1D1*36 C>T (rs1872930) allelic variant is associated with variability of the CYP2C9 genotype predicted pharmacokinetics of ibuprofen enantiomers - a pilot study in healthy volunteers, Acta Pharm. 69 (2019) $399-412$.

Rev. London. January 2010) and EMA Ibuprofen oral use immediate release formulations 200-800 mg product-specific bioequivalence guidance (EMA/CHMP/356876/2017).

None of the participants were related; they were fully informed about the nature of both PK and pharmacogenetic (PGx) studies, gave their written informed consent and could discontinue their participation at any time during the trials. None of the subjects participated in more than one trial. The exclusion criteria of interest were clinically significant illnesses and surgery within 4 weeks prior to the first dosing; clinically significant abnormalities of medical history (neurological, endocrinal, cardiovascular, pulmonary, hematological, immunologic, psychiatric, gastrointestinal, renal, hepatic, and metabolic diseases) at physical examination, of the ECG, of hematology, biochemistry or urinalysis results; history of hypersensitivity reactions (e.g. asthma, rhinitis, angioedema, or urticaria) in response to aspirin or other NSAIDs; history of recurrent peptic ulcer/hemorrhage; history of GI disease (ulcerative colitis, Crohn's disease); history of GI bleeding or perforation related to previous NSAID therapy; positive alcohol test at Check-in (Day-1) and history of alcohol and/or drug abuse within one year prior to the screening; use of investigational drugs within 28 days (4 weeks) or any drugs known to induce or inhibit hepatic drug metabolism within 28 days ( 4 weeks) prior to the first administration of the study medication and participation in other clinical studies within 2 months before the study.

\section{Bioequivalence trials design and procedures}

All four IBP bioequivalence trials were designed as single-center, randomized, open-label, two-way cross-over bioequivalence studies of the oral, immediate-release formulation of IBP with systemic action (with identical contents of both enantiomers), following a single dose administration under fasting conditions. The IBP plasma concentrations of 12 subjects receiving $400 \mathrm{mg}$, 10 subjects participating in the studies with $375 \mathrm{mg}$, 2 subjects receiving $200 \mathrm{mg}$ and 3 subjects receiving $100 \mathrm{mg}$ oral IBP were used in the PK analysis. According to the study protocols, a total of 20 blood samples were collected in each period: pre-dose and $0.083,0.167,0.333,0.5,0.667,0.833,1,1.33,1.67,2,2.5,3,4,5,6,8,10,12$, and 14 hours post-dose. An additional blood sample was collected at Check-in (Day-1) of Period 1 for the investigation of potential pre-dose concentrations and this sample was not used for pharmacokinetic evaluation. The subjects remained at the clinical unit during the whole period, i.e. until the collection of the $14 \mathrm{~h}$ post drug blood sample. The samples were centrifuged at $4{ }^{\circ} \mathrm{C}$ for $10 \mathrm{~min}$ at $3500 \mathrm{rpm}$ and plasma aliquots (labeled with the protocol code, the subject number, the period and the time at which the sample was obtained) were stored at $-30^{\circ} \mathrm{C}$ until further analytic determinations.

\section{Pharmacokinetic analysis}

Concentrations of $R-(-)$-IBP and $S$-(+)-IBP were determined using validated high-performance liquid chromatography coupled with a tandem mass spectrometry detection (HPLC MS/MS) chiral method according to Nakov et al. (23). Estimation of the PK parameters was performed from the plasma concentration-time dependent data by a standard non-compartmental method using Pharsight ${ }^{\circledR}$ Knowledgebase ServerTM and Phoenix ${ }^{\circledR}$ WinNonlin ${ }^{\circledR}$. Maximum plasma concentration $\left(c_{\max }\right)$ and $t_{\max }$ were obtained directly from raw data. The area under the curve to the last measured concentration $\left(A U C_{0-t}\right)$ was calcu- 
lated by linear trapezoidal integration. The area under the curve extrapolated to infinity $\left(A U C_{0-\infty}\right)$ was calculated as the sum of $A U C_{0-\mathrm{t}}$ and the residual area $\left(c_{\mathrm{t}}\right.$ divided by $K_{\mathrm{e}}$, with $c_{\mathrm{t}}$ as the last measured concentration and $K_{\mathrm{el}}$ as the elimination rate constant). Because of different doses administered in different trials, AUC and $c_{\max }$ were divided by the dose according to the body weight $\left(\mathrm{mg} \mathrm{kg}^{-1}\right)$ of each subject. The elimination half-life $\left(t_{1 / 2}\right)$ was calculated as $0.693 \mathrm{~K}_{\mathrm{el}}^{-1}$. Total drug clearance adjusted for bioavailability $\left(\mathrm{Cl}_{\mathrm{tot}}\right)$ was calculated by dividing the dose by $A U C$ and weight-adjusting.

\section{CYP2C9 and AKR1D1 genotyping}

Genomic DNA was isolated from $3 \mathrm{~mL}$ whole blood (collected in an EDTA blood tube) using proteinase $\mathrm{K}$ digestion/phenol-chloroform extraction and ethanol precipitation and stored at $4{ }^{\circ} \mathrm{C}$ until genotyping. The obtained DNA was spectrophotometrically quantified on Nano Drop ${ }^{\circledR}$ and its purity was verified using a $260 / 280 \mathrm{~nm}$ ratio. DNA integrity was confirmed by $0.8 \%$ agarose electrophoresis, stained with ethidium bromide. Identification of CYP2C9*2 (rs1799853), CYP2C9*3 (rs1057910) and AKR1D1*36C >T (rs1872930) variant alleles was done with a real-time polymerase chain reaction [MxPro 3005P, Stratagene, La Jolla, CA, USA] using TaqMan SNP and drug metabolism genotyping assays according to the manufacturers' instructions [Applied Biosystems, Foster City, CA, USA]. The CYP2C9 and AKR1D1 genotyping was performed at the Center for Biomolecular and Pharmaceutical Analysis, Faculty of Pharmacy, University "Ss Cyril and Methodius" in Skopje, Macedonia.

\section{Statistical analysis}

The Hardy-Weinberg equilibrium was estimated for all analyzed variants. Deviations from the equilibrium were detected by comparing the observed and expected frequencies using Fisher's exact test. Pearson's chi-square test was used for evaluation of differences in the CYP2C9 and AKR1D1 allelic and genotypic frequencies between the study subjects and those reported for the Macedonian population. Values of PK parameters were expressed as mean \pm SD. All statistical analyses were performed using MedCalc 14.12.0 and StatGraph17.1.03 software packages. Differences in PK parameters among individuals with different genotypes were assessed with the Kruskal-Wallis test and Dunn test for post hoc analysis. Odds ratios [OR] were calculated with $95 \%$ confidence limits [95\% CI]. Factors with $p \leq 0.05$ were considered statistically significant.

\section{RESULTS AND DISCUSION}

\section{Pharmacokinetic parameters}

Interindividual variability in the PK parameters for IBP enantiomers, present at identical concentrations in the administered racemic product, was investigated in 27 healthy volunteers. Mean values of the PK parameters are summarized in Table I. Although the mean $A U C_{0 \text {-inf }}$ for $S-(+)$-IBP was slightly higher compared to the mean value for $R-(-)$-IBP (49.985 $\mathrm{mg} \mathrm{h} \mathrm{L}^{-1}$ vs. $42.606 \mathrm{mg} \mathrm{h} \mathrm{L}^{-1}$, respectively), we could not confirm the one-way chiral conversion of $R-(-)$ to $S-(+)$ from previously published data (5), most probably because of the small sample size. High interindividual variability for both $S-(+)$ and $R-(-)-$ IBP regard- 
A. Kapedanovska Nestorovska et al.: AKR1D1*36 C>T (rs1872930) allelic variant is associated with variability of the CYP2C9 genotype predicted pharmacokinetics of ibuprofen enantiomers - a pilot study in healthy volunteers, Acta Pharm. 69 (2019) 399-412.

Table I. Summary of pharmacokinetic parameters for S-(+) and R-(-)-IBP enantiomers in healthy volunteers

\begin{tabular}{lcccc}
\hline \multirow{2}{*}{ PK parameters } & \multicolumn{3}{c}{ Ibuprofen enantiomers $(N=27)$} \\
\cline { 2 - 5 } & \multicolumn{2}{c}{$S-(+)$-IBP } & \multicolumn{2}{c}{$R$-(-)-IBP } \\
\cline { 2 - 5 } & Mean $\pm \mathrm{SD}$ & $95 \% \mathrm{CI}$ & $95 \% \mathrm{CI}$ \\
\hline$A U C_{0-\mathrm{t}}\left(\mathrm{mgh} \mathrm{L}^{-1}\right)$ & $48.335 \pm 19.854$ & $44.512-52.159$ & $42.173 \pm 17.861$ & $38.733-45.613$ \\
$A U C_{0-\infty}\left(\mathrm{mg} \mathrm{h} \mathrm{L}^{-1}\right)$ & $49.985 \pm 21.408$ & $45.862-54.108$ & $42.606 \pm 17.904$ & $39.157-46.054$ \\
$C_{\text {tot }}\left(\mathrm{L} \mathrm{h}^{-1}\right)$ & $7.219 \pm 2.824$ & $6.675-7.763$ & $8.460 \pm 3.210$ & $7.842-9.078$ \\
$C_{\text {max }}\left(\mathrm{mg} \mathrm{L}^{-1}\right)$ & $12.691 \pm 3.939$ & $11.932-13.449$ & $13.108 \pm 4.439$ & $12.253-13.963$ \\
$K_{\mathrm{el}}\left(\mathrm{L} \mathrm{h}^{-1}\right)$ & $0.276 \pm 0.0351$ & $0.269-0.283$ & $0.403 \pm 0.105$ & $0.383-0.424$ \\
$t_{\max }(\mathrm{h})$ & $2.558 \pm 0.379$ & $2.485-2.631$ & $0.965 \pm 0.669$ & $0.836-1.094$ \\
$t_{1 / 2}(\mathrm{~h})$ & $1.089 \pm 0.691$ & $0.956-1.223$ & $1.856 \pm 0.573$ & $1.745-1.966$ \\
\hline
\end{tabular}

a)

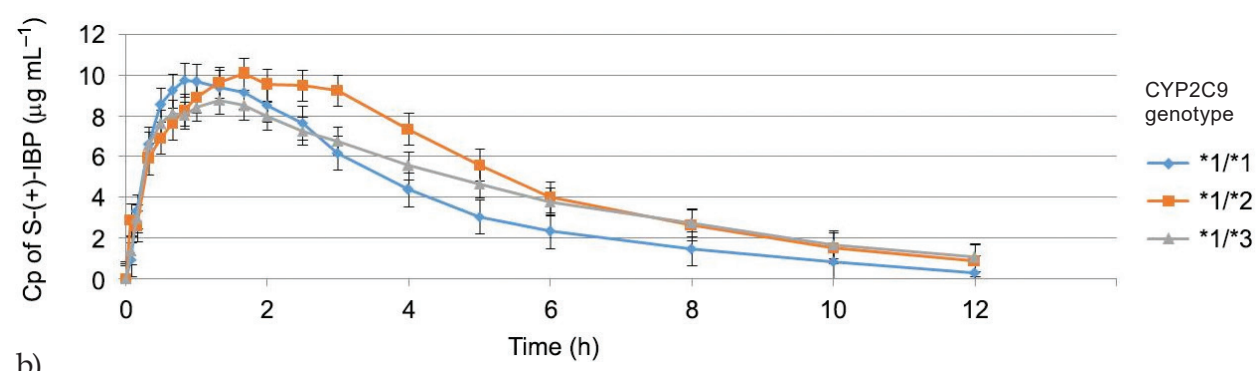

b)

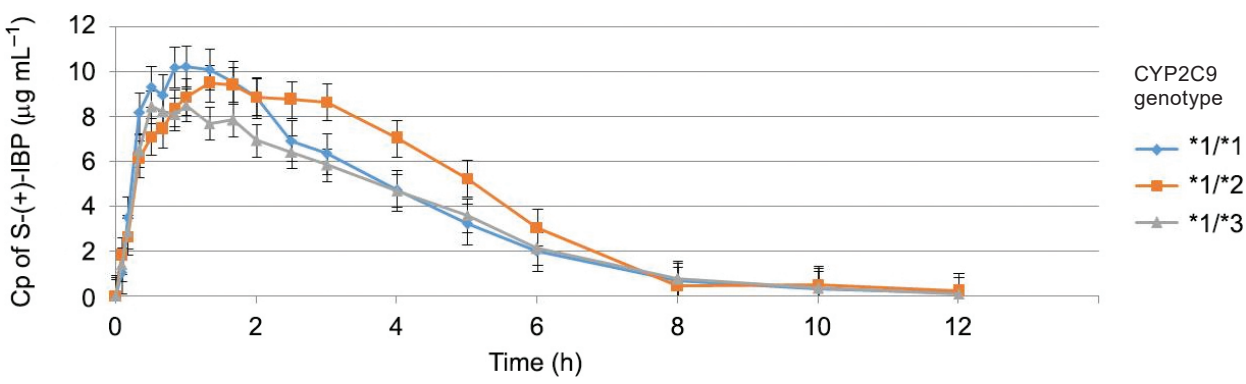

Fig. 1. Time dependent, dose corrected plasma concentrations of a) $S$-(+)-IBP and b) $R-(-)$-IBP after administration of single dose racemic IBP in 27 healthy volunteers stratified according to the CYP2C 9 genotype.

ing $C l_{\text {tot }}$ and $c_{\max }$ was observed. Variation of $C l_{\text {tot }}$ and $c_{\max }$ (interval between the minimal and maximal value) for $S$-(+)-IBP was $2.56-13.47 \mathrm{~L} \mathrm{~h}^{-1}$ and $5.36-24.35 \mathrm{mg} \mathrm{L}^{-1}$; while it was 3.74-16.70 $\mathrm{L} \mathrm{h}^{-1}$ and 1.07-22.01 $\mathrm{mg} \mathrm{L}^{-1}$, respectively, for $R$-(-)-IBP. As a result, high interindividual variability was also observed for $A U C_{0 \text {-inf }}$ ranging between $25.38-109.93 \mathrm{mg} \mathrm{h} \mathrm{L}^{-1}$ or $S-(+)-I B P$ and $16.65-76.87 \mathrm{mg} \mathrm{h} \mathrm{L}^{-1}$ for $R-(-)-I B P$. 


\section{Genotyping for CYP2C9 and AKR1D1 alleles}

CYP2C9 and AKR1D1 allelic frequencies and genotype distributions in the considered population of healthy volunteers $(N=27$ subjects) are presented in Table II. All investigated genetic variants (CYP2C9*2, CYP2C9*3 and AKR1D1*36C>T) were in Hardy-Weinberg equilibrium ( $p=0.558, p=0.639, p=0.936$, respectively) and their frequencies were in accord with those reported for the population of The Republic of Macedonia $(p=0.342$ for CYP2C9*2, $p=1$ for CYP2C9*3 and $p=0.664$ for AKR1D1*36C $>$ T) $(24,25)$. No homozygosity for the variant CYP2C9 $* 2$ and $* 3$ alleles $(* 2 / 2$ and $* 3 / 3$ genotype) was detected and none of the subjects was identified as a carrier of both CYP2C9 variant alleles $\left({ }^{*} 2 /{ }^{*} 3\right.$ genotype) in this study. This finding was expected taking into account the small sample size as well as the fact that the prevalence of these functionally significant genotypes in most populations, including the Macedonian, is below 0.05 .

\section{Evaluation of CYP2C9 PGx/PK relationship}

The time dependent plasma concentration curves of both IBP enantiomers according to the CYP2C9 genotype are shown in Fig. 1. The observed effects were similar to previously published reports $(9,26-28)$, suggesting that both CYP2C9 *2 and *3 alleles reduce enzyme activity. Pharmacokinetic parameters derived from a non-compartmental analysis of individual data are presented in Table III. Significant differences across the three genotype groups regarding S-(+)-IBP for $A U C_{0-\mathrm{t}}(p=0.016), A U C_{0-\infty}(p=0.019), C l_{\text {tot }}(p=0.022)$ and $c_{\max }(p=0.039)$ were observed. Pairwise comparisons revealed that the subjects with the CYP2C9 ${ }^{*} 1 /{ }^{*} 2$ genotype had $25 \%$ lower $C l_{\text {tot }}$ and $14 \%$ higher $c_{\max }$ compared to subjects with the CYP2C9 ${ }^{*} 1 /{ }^{*} 1$ CYP2C9 $* 1 /{ }^{*} 3$ genotype. CYP2C $9{ }^{*} 1 /{ }^{*} 2$ had markedly higher $A U C_{0-t}$ and $A U C_{0-\infty}$ values (25\% higher) than those found for CYP2C9*1/*1.

In case of $R$-(-)-IBP, significant differences across the three genotype groups regarding $C l_{\text {tot }}(p=0.001), c_{\max }(p=0.023), K_{\mathrm{el}}(p=0.024)$ and $t_{1 / 2}(p=0.024)$ were identified. Pairwise

Table II. Genotype distribution and allelic frequencies of CYP2C9 and AKR1D1genetic variants in the study population of healthy volunteers

\begin{tabular}{|c|c|c|c|c|c|c|c|}
\hline & \multirow{2}{*}{ Genotype } & \multirow{2}{*}{$n$} & \multicolumn{2}{|c|}{ Frequencies } & \multirow{2}{*}{ Allele } & \multirow{2}{*}{$n$} & \multirow{2}{*}{ Frequencies } \\
\hline & & & observed & expected & & & \\
\hline \multirow{6}{*}{ СУР2C9 } & ${ }^{*} 1 /{ }^{*} 1$ & 18 & 0.667 & 0.694 & \multirow{2}{*}{${ }^{*} 1$} & \multirow{2}{*}{45} & \multirow{2}{*}{0.833} \\
\hline & $* 1 / * 2$ & 5 & 0.185 & 0.154 & & & \\
\hline & ${ }^{*} 1 /{ }^{* 3}$ & 4 & 0.148 & 0.123 & \multirow{2}{*}{ *2 } & \multirow{2}{*}{5} & \multirow{2}{*}{0.093} \\
\hline & $* 2 / * 2$ & 0 & 0.000 & 0.009 & & & \\
\hline & $* 3 / * 3$ & 0 & 0.000 & 0.005 & \multirow{2}{*}{ *3 } & \multirow{2}{*}{4} & \multirow{2}{*}{0.074} \\
\hline & ${ }^{*} 2 / * 3$ & 0 & 0.000 & 0.014 & & & \\
\hline \multirow{3}{*}{ AKR1D1 } & ${ }^{*} 1 /{ }^{*} 1$ & 14 & 0.519 & 0.522 & \multirow{2}{*}{${ }^{*} 1$} & \multirow{2}{*}{39} & \multirow{2}{*}{0.722} \\
\hline & $* 1 / * 36$ & 11 & 0.407 & 0.401 & & & \\
\hline & $* 36 / * 36$ & 2 & 0.074 & 0.077 & $* 36$ & 15 & 0.278 \\
\hline
\end{tabular}




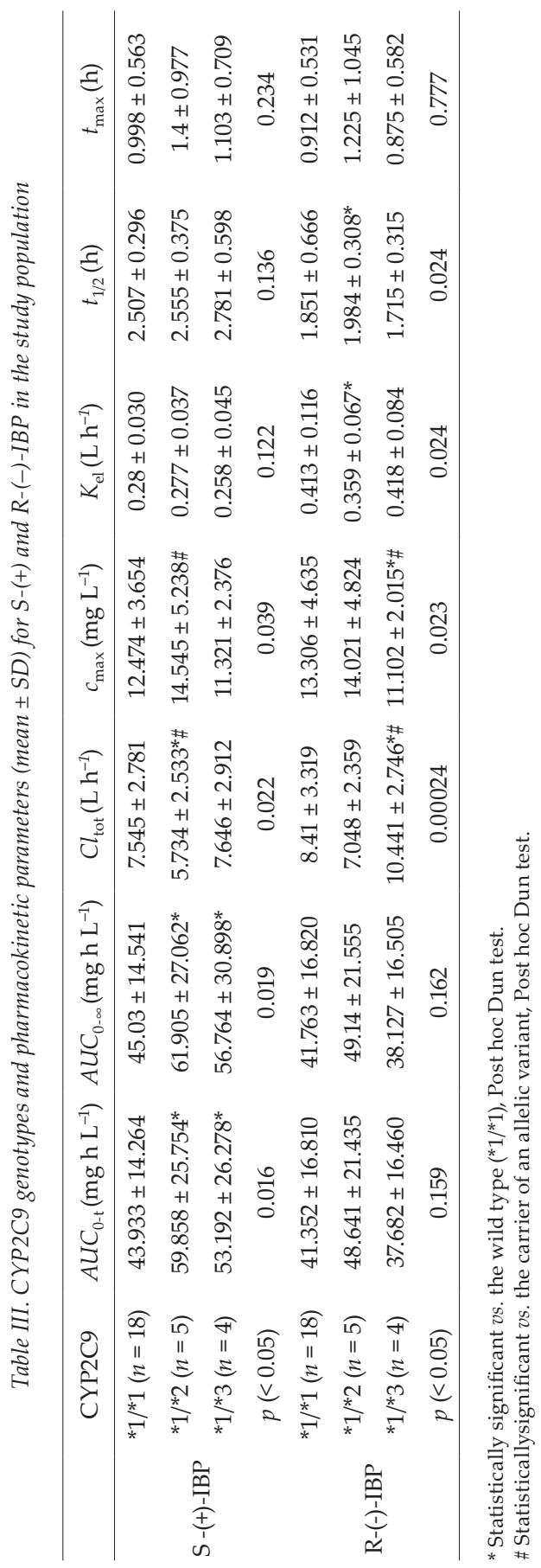

comparisons revealed that the heterozygote carriers of CYP2C 9 *3 allele had a 16 $\%$ increase in $\mathrm{Cl}_{\text {tot }}$ and $21 \%$ decrease in $c_{\max }$ in comparison with the carriers of CYP2C $9 * 2$ or CYP2C ${ }^{*} 1 /{ }^{*} 1$ genotype. In addition, the CYP2C9 $* 2$ allele was associated with $25 \%$ higher $K_{\mathrm{el}}$ and $10 \%$ lower $t_{1 / 2}$ values compared to subjects carrying the CYP2C $9{ }^{*} 1 /{ }^{*} 1$ genotype. For both IBU enantiomers, $t_{\max }$ was the only pharmacokinetic parameter not associated with any of the CYP2C9 polymorphic alleles. Based on the results of our research, both CYP2C9 *2 and *3 polymorphisms have to be considered as factors associated with a change in PK parameters of IBP. Still, unlike other studies $(9,16,27)$, our data suggest a stronger impact on IBP PK for CYP2C $9 * 2$ compared to the CYP2C9*3 allele. This might be attributed to the underrepresentation of homozygotes for the variant alleles $(* 2 / * 2 ; * 3 / * 3$ and $* 2 / * 3$ genotypes) considering that changes in PK parameters are proportional to the number of CYP2C9 variant alleles (20). Moreover, they suggest that even though CYP2C9 is considered a major genetic factor for $R-(-)$ and $S-(+)-I B P$ clearance, the effect of CYP2C8 should also be measured since CYP2C8*3 alone or in combination with CYP2C9 *2 has a more significant impact on IBP AUC and clearance than CYP2C9 *3 $(26,27,29,30)$. Bearing in mind the high sequence homology between CYP2C9 and CYP2C8 as well as the recognized $\mathrm{LD}$ between the $\mathrm{CY}$ $\mathrm{P} 2 \mathrm{C}^{*} 3$ and CYP2C9*2 alleles; where 96 $\%$ of the subjects with the CYP2C $8 * 3$ allele also carry CYP2C9*2 and $85 \%$ of the subjects with CYP2C9*2 variant also have a $\mathrm{CYP} 2 \mathrm{C} 8^{*} 3(17,18)$, we did not assess the effect of CYP2C8 polymorphism on IBP enantiomeric metabolism. However, the results obtained are in agreement with those previously reported by Rodrigues and Rushmore (31), which proved that when evaluating only the 
A. Kapedanovska Nestorovska et al.: AKR1D1*36 C>T (rs1872930) allelic variant is associated with variability of the CYP2C9 genotype predicted pharmacokinetics of ibuprofen enantiomers - a pilot study in healthy volunteers, Acta Pharm. 69 (2019) $399-412$.

CYP2C9 genotype status, there is no significant difference in IBP PK parameters between subjects genotyped as CYP2C $9{ }^{*} 1 /{ }^{*} 3$ and ${ }^{*} 1 /{ }^{*} 1$.

Although the differences observed in PK parameters, especially clearance, after oral administration in subjects with different CYP2C9 genotypes (dominantly carriers of CY$\mathrm{P} 2 \mathrm{C} 9 * 2 / * 2 ; * 2 * 3$ and $* 3 / * 3$ ), suggest that the safety and efficacy of CYP2C9 substrates could be improved by drug dose adjustment $(32,33)$ and since the correlation between the genotype and phenotype is not clear, prospective evaluations of this strategy are needed before establishing its clinical utility.

\section{Evaluation of AKR1D1 PGx/PK relationship}

Data regarding the impact of the AKR1D1 genetic variant on the IBP enantiomeric metabolism are summarized in Table IV. Careers of *36 allele had significantly higher $C l_{\text {tot }}$ and notably shorter $t_{\max }$ for both IBU enantiomers compared to wild type carriers $(p<0.001)$. Furthermore, AKR1D1 *36 allele was associated with significantly lower $A U C_{(0-t) \text { and } 0-\infty)}$ values but only in regard to $S-(+)-I B P$. We observed that the increased AKR1D1 enzyme activity in the metabolic biotransformation of IBU enantiomers was not proportional to the number of *36 variant alleles. For $S$-(+)-IBP, the genotype model of analysis showed significant differences across the three genotype groups regarding $A U C_{0-\mathrm{t}}$ and $A U C_{0-\infty}\left(p<0.001\right.$, for all), $C l_{\text {tot }}$ and $t_{\max }(p<0.001)$. The post hoc Dunn test revealed that the AKR1D1 ${ }^{*} 1 /{ }^{*} 36$ genotype was associated with higher $C l_{\text {tot }}$ and shorter $t_{\max }$ values compared to the AKR1D1*36/*36 genotype and lower AUC $\left({ }_{0-t}\right.$ and $\left.{ }_{0-\infty}\right)$ values than those found for AKR1D1 ${ }^{*} 1 /{ }^{*} 1$ and AKR1D1 $* 36 / * 36$ genotypes. The same pattern of significant alterations $(p<0.001)$ regarding $A U C_{(0-t)}$ and ${ }_{0-\infty)}, C l_{\text {tot }}$ and $t_{\max }$ across the three genotypes was observed for $R-(-)$-IBP.

Since we had established previously that CYP2C9 $* 2$ had a marked effect on IBP PK while the effect of *3 allele was indistinct, we supposed that the CYP2C9 genotype confounded the association between AKR1D1*36 and IBP PK parameters. Therefore, an additional analysis was performed to assess the impact of the AKR1D1 *36 variant allele but only in subjects who are not carriers of the CYP2C9 *2 allele (Table V). In these subjects, the carriers of AKR1D1*36 allele had significantly lower $A U C\left({ }_{0-t}\right.$ and $\left.0-\infty\right)$, higher $C l_{\text {tot }}$ and shorter $t_{\max }$ values for both $S-(+)$-IBP and $R$-(-)-IBP. Interestingly, in this sub-stratification analysis, the differences for IBP PK parameters were proportional to the number of the AKR1D1*36 alleles. A statistically significant variation across the three genotypes regarding $A U C\left(_{0-t}\right.$ and $\left.0-\infty\right)$ $C l_{\text {tot }}$ and $t_{\max }(p<0.001)$ was found for both IBP enantiomers. Pairwise comparisons showed that the AKR1D1 *36/*36 genotype was associated with increased metabolic biotransformation of IBP compared to other AKR1D1 genotypes. These results confirmed our initial assumption regarding the clear influence of the CYP2C9*2 allelic variant on IBP PKs and highlights the distinct role of AKR1D1*36C $>\mathrm{T}$ in the metabolism of IBP.

The present study is the first to investigate the relationship between AKR1D1*36C>T (rs1872930) and the combined AKR1D1/CYP2C9 effect on the PK of IBP enantiomers. Regarding the contribution to previous knowledge $(19,21)$ and our expectations, it is still unclear whether the AKR1D1*36 C>T (rs1872930) allelic variant influences CYP2C9 enzyme activity (i.e. catalytic activity, kinetics), or the expression of CYP2C9 mRNA, and consequently enzyme activity. Future studies specifically designed to answer this questions of broader biological relevance would be very interesting. However, the results obtained in the present study support the data published by Chaundry et al. (21) and Tracy et al. (19) 


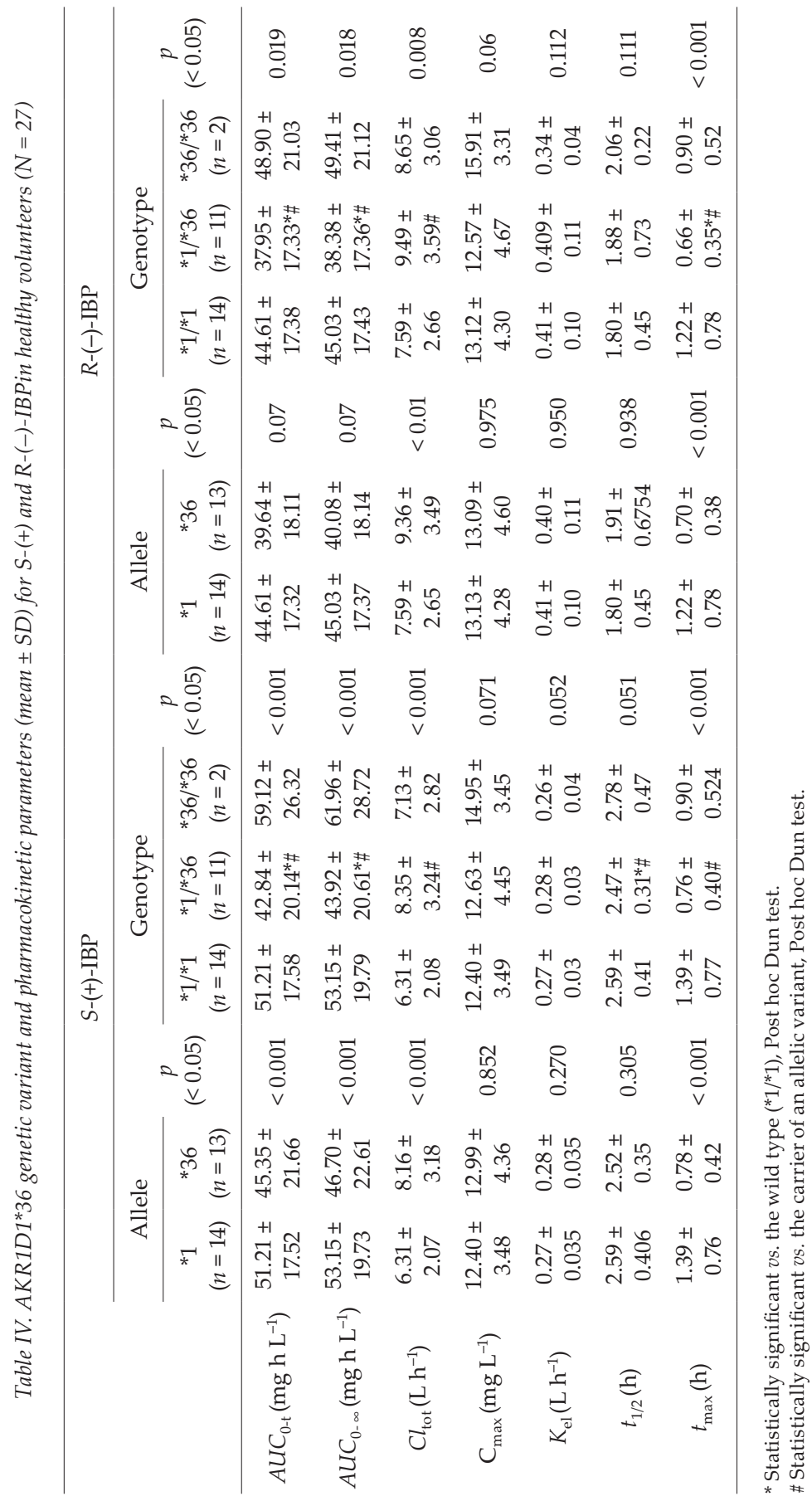




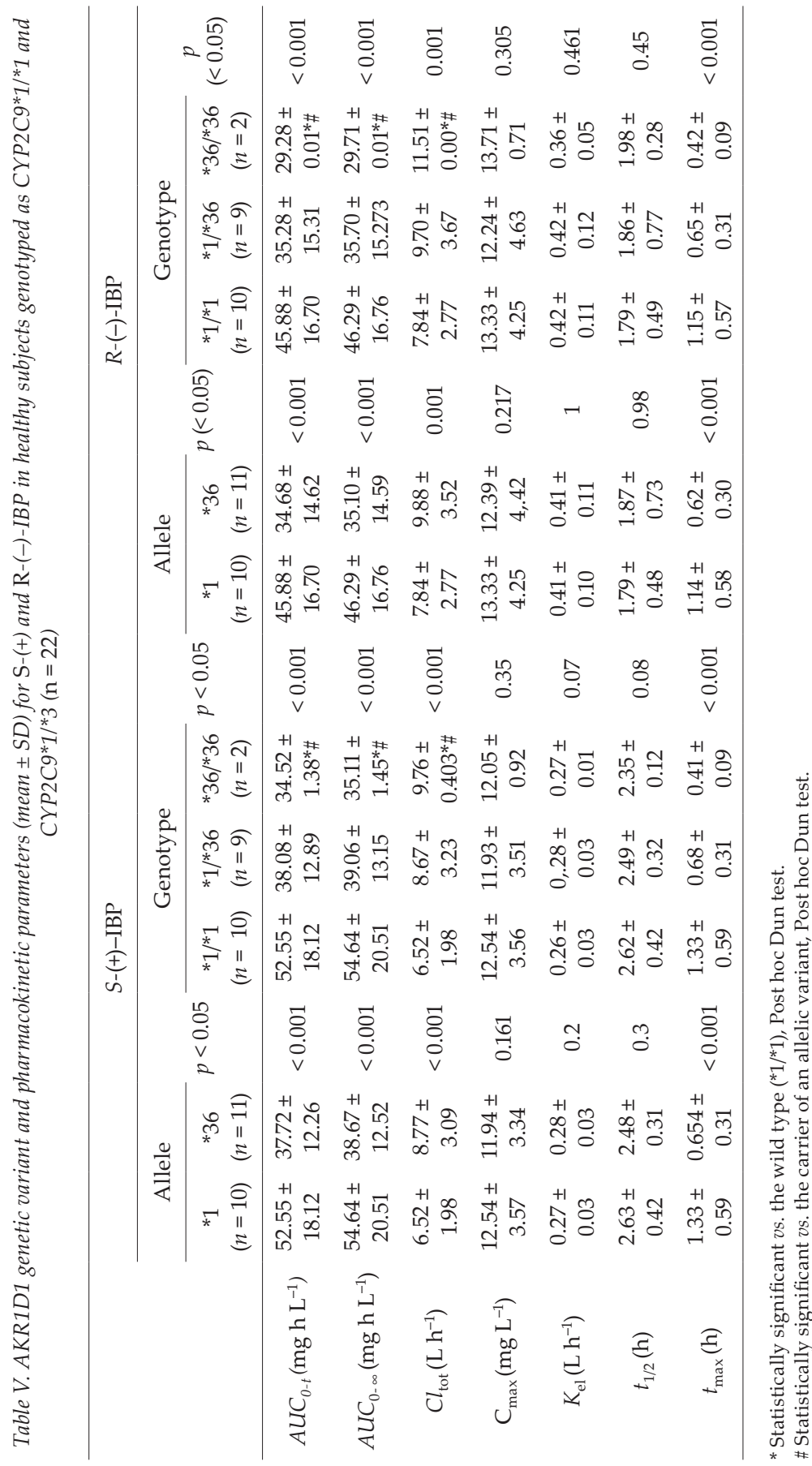


A. Kapedanovska Nestorovska et al.: AKR1D1*36 C>T (rs1872930) allelic variant is associated with variability of the CYP2C9 genotype predicted pharmacokinetics of ibuprofen enantiomers - a pilot study in healthy volunteers, Acta Pharm. 69 (2019) $399-412$.

and add to the evidence that genetic variation in genes, like AKR1D1, is an important contributor to variation in CYP450 activity, by influencing the level of ligands that activate nuclear receptors responsible for the transcriptional regulation of P450s (e.g., bile acids).

In this report, we provide evidence for the association between CYP2C9 cis-allelic variants, AKR1D1 trans-genetic regulator of CYP450 gene network and the PK profile of IBP enantiomers. A crucial observation from the study is the association between the AKR1D1 minor allele and the increased IBP metabolism in subjects who are carriers of CYP2C ${ }^{*} 1 /{ }^{*} 3$ or CYP2C 9 wild genotype. Our model implies that patients on a long-term IBP treatment who are not carriers of CYP2C9*2 allele require additional stratification according to the AKR1D1 genotype in order to avoid the risk of adverse IBP side events. A potential weakness of the current study is the small number of subjects. In the next phase of the project, we will further evaluate the value of concomitant CYP2C9 and AKR1D1 genotyping and conduct a reanalysis in a larger cohort of subjects with a higher representation of $\mathrm{CYP} 2 \mathrm{C} 8 / \mathrm{C} 9$ genotypes.

\section{CONCLUSSIONS}

The results from this pilot study contribute to the efforts aimed at clearer elucidation of the ibuprofen metabolic phenotype. They suggest that significant changes in the PK values associated with reduced CYP2C9 enzyme activity are anticipated to occur only in the carriers of the CYP2C9*2 allelic variant. AKR1D1*36 provides a new insight regarding the interindividual differences in the metabolism of the IBP enantiomers. The value of concomitant CYP2C9 and AKR1D1 genotyping has to be further proven in studies with a larger sample size and in prospective randomized clinical studies.

Acknowledgments. - The authors are grateful to N. Geskovski and N. Labacevski for their critical reviewing of earlier versions of the manuscript and for valuable suggestions and comments. The study would not have been possible without the cooperation of healthy volunteers.

\section{REFERENCES}

1. K. D. Rainsford, Fifty years since the discovery of ibuprofen, Inflammopharmacol. 19 (2011) 293-297; https://doi.org/10.1007/s10787-011-0103-7

2. M. De Martino, A. Chiarugi, A. Boner, G. Montini and L. de' Angelis, Working towards an appropriate use of ibuprofen in children: An evidence-based appraisal, Drugs 77 (2017) 1295-1311; https://doi.org/10.1007/s40265-017-0751-z.

3. H. Hao, G. Wang and Sun, Enantioselective pharmacokinetics of ibuprofen and involved mechanisms, Drug Metab. Rev. 37 (2005) 215-234; https://doi.org/10.1081/DMR-200047999

4. K. D. Rainsford, Ibuprofen: pharmacology, efficacy and safety, Inflammopharmacol. 17 (2009) 275342; https://doi.org/10.1007/s10787-009-0016-x

5. G. Ding, Y. Liu, J. Sun, Y. Takeuchi, T. Toda, T. Hayakawa, S. Fukushima, S. Kishimoto, W. Lin and Inotsume, Effect of absorption rate on pharmacokinetics of ibuprofen in relation to chiral inversion in humans, J. Pharm. Pharmacol. 59 (2007) 1509-13; https://doi.org/10.1211/jpp.59.11.0007

6. T. J. Legg, A. L. Laurent, R. Leyva and D. Kellstein, Ibuprofen sodium is absorbed faster than standard Ibuprofen tablets: results of two open-label, randomized, crossover pharmacokinetic studies, Drugs R.D. 14 (2014) 283-290; https://doi.org/10.1007/s40268-014-0070-8 
A. Kapedanovska Nestorovska et al.: AKR1D1*36 C>T (rs1872930) allelic variant is associated with variability of the CYP2C9 genotype predicted pharmacokinetics of ibuprofen enantiomers - a pilot study in healthy volunteers, Acta Pharm. 69 (2019) 399-412.

7. F. Jamali and D. R. Brocks, The Pharmacokinetics of Ibuprofen in Humans and Animals, in Ibuprofen (Ed. K. Rainsford), $1^{\text {st }}$ ed., John Wiley \& Sons, New York 2015, pp. 81-131; https://doi.org/10.1002/ 9781118743614.ch4

8. S. C. Tan, B. K. Patel, S. H. Jackson, C. G. Swift and A. J. Hutt, Stereoselectivity of ibuprofen metabolism and pharmacokinetics following the administration of the racemate to healthy volunteers, Xenobiotica 32 (2002) 683-697; https://doi.org/10.1080/00498250210142994

9. J. Kirchheiner, I. Meineke, G. Freytag, C. Meisel, I. Roots and J. Brockmöller, Enantiospecific effects of cytochrome P450 2C9 amino acid variants on ibuprofen pharmacokinetics and on the inhibition of cyclooxygenases 1 and 2, Clin. Pharmacol. Ther. 72 (2002) 62-75; https://doi.org/10.1067/ mcp.2002.125726

10. S. Y. Chang, W. Li, S. C. Traeger, B. Wang, D. Cui, H. Zhang, B. Wen and A. D. Rodrigues, Confirmation that cytochrome P450 2C8 (CYP2C8) plays a minor role in (S)-(+)- and (R)-(-)-ibuprofen hydroxylation in vitro, Drug Metab. Dispos. 36 (2008) 2513-2522; https://doi.org/10.1124/ dmd.108.022970

11. A. D. Rodrigues, Impact of CYP2C9 genotype on pharmacokinetics: Are all cyclooxygenase inhibitors the same? Drug Metab. Dispos. 33 (2005) 1567-1575; https://doi.org/10.1124/dmd.105.006452

12. F. Mazhar, N. Haider, J. Sultana, S. Akram and Y. Ahmed, Prospective study of NSAIDs prescribing in Saudi Arabia: Cardiovascular and gastrointestinal risk in patients with diabetes mellitus, Int. J. Clin. Pharmacol. Ther. 56 (2018) 64-71; https://doi.org/10.5414/CP203071

13. O. O. Moninuola, W. Milligan, P. Lochhead and H. Khalili, Systematic review with meta-analysis: association between acetaminophen and nonsteroidal anti-inflammatory drugs (NSAIDs) and risk of Crohn's disease and ulcerative colitis exacerbation, Aliment. Pharmacol. Ther. 47 (2018) 14281439; https://doi.org/10.1111/apt.14606

14. A. Pilotto, D. Seripa, M. Franceschi, C. Scarcelli, D. Colaizzo, E. Grandone, V. Niro, A. Andriulli, G. Leandro, F. Di Mario and B. Dallapiccola, Genetic susceptibility to nonsteroidal anti-inflammatory drug-related gastroduodenal bleeding: role of cytochrome P450 2C9 polymorphisms, Gastroenterology 133 (2007) 465-471; https://doi.org/10.1124/dmd.105.006452

15. G. S. Pazhayattil and A. C. Shirali, Drug-induced impairment of renal function, Int. J. Nephrol. Renovasc. Dis. 7 (2014) 457-468, https://doi.org/10.2147/IJNRD.S39747

16. G. V. Rollason, C. F. Samer, Y. Daali and J. A. Desmeules, Prediction by pharmacogenetics of safety and efficacy of non-steroidal anti-inflammatory drugs: a review, Curr. Drug Metab. 15 (2014) 326-343; https://doi.org/10.2174/1389200215666140202214454

17. U. Yasar, S. Lundgren, E. Eliasson, A. Bennet, B. Wiman, U. de Faire and A. Rane, Linkage between the CYP2C8 and CYP2C9 genetic polymorphisms, BiochemBiophysResCommun. 299 (2002) 25-28; https://doi.org/10.1016/S0006-291X(02)02592-5

18. W. C. Speed, S. P. Kang, D. P. Tuck, L. N. Harris and K. K. Kidd, Global variation in CYP2C8-CYP2C9 functional haplotypes, Pharmacogenomics J. 9 (2009) 283-290; https://doi.org/10.1038/ tpj. 2009.10

19. T. S. Tracy, A. S. Chaudhry, B. Prasad, K. E. Thummel, E. G. Schuetz, X. B. Zhong, Y. C. Tien, H. Jeong, X. Pan, L. M. Shireman, J. Tay-Sontheimer and Y. S. Lin, Interindividual variability in cytochrome P450-mediated drug metabolism, Drug Metab. Dispos. 44 (2016) 343-351; https://doi. org/10.1124/dmd.115.067900

20. J. Kirchheiner and J. Brockmöller, Clinical consequences of cytochrome P4502C9 polymorphisms, Clin. Pharmacol. Ther. 77 (2005) 1-16; https://doi.org/10.1016/j.clpt.2004.08.009

21. A. S. Chaudhry, R. K. Thirumaran, K. Yasuda, X. Yang, Y. Fan, S. C. Strom and E. G. Schuetz, Genetic variation in aldo-keto reductase 1D1 (AKR1D1) affects the expression and activity of multiple cytochrome P450s, Drug Metab. Dispos. 41 (2013) 1538-1547; https://doi.org/10.1124/ dmd.113. 051672 
A. Kapedanovska Nestorovska et al.: AKR1D1*36 C>T (rs1872930) allelic variant is associated with variability of the CYP2C9 genotype predicted pharmacokinetics of ibuprofen enantiomers - a pilot study in healthy volunteers, Acta Pharm. 69 (2019) 399-412.

22. T. M. Penning, The aldo-keto reductases (AKRs): Overview, Chem. Biol. Interact. 234 (2015) 236-246; https://doi.org/10.1016/j.cbi.2014.09.024

23. N. Nakov, L. Bogdanovska, J. Acevska, J. Tonic-Ribarska, R. Petkovska A. Dimitrovska, L. Kasabova and D. Svinarov, High-throughput HPLC-MS/MS method for quantification of ibuprofen enantiomers in human plasma: Focus on investigation of metabolite interference, J. Chromatogr. Sci. 54 (2016) 1820-1826; https://doi.org/10.1093/chromsci/bmw166

24. K. Jakovski, A. Kapedanovska Nestorovska, N. Labacevski and A. J. Dimovski, Characterization of the most common CYP2C9 and CYP2C19 allelic variants in the population of R. Macedonia, Pharmazie 68 (2013) 893-898, https://doi.org/10.1691/ph.2013.3579

25. A. Kapedanovska Nestorovska, K. Jakovski, Z. Naumovska, Z. Sterjev, N. Matevska Geskovska, A. J. Dimovski and Lj. Suturkova, Allele frequency and genotype distribution of aldo keto reductase 1D1 (AKR1D1) rs1872930 genetic variant in a Macedonian population, Maced. Pharm. Bull. 64 (2018), in press.

26. C. Martínez, E. García-Martín, G. Blanco, F. J. Gamito, J. M. Ladero and J. A. Agúndez, The effect of the cytochrome P450 CYP2C8 polymorphism on the disposition of $(R)$-ibuprofen enantiomer in healthy subjects, Br. J. Clin. Pharmacol. 59 (2005) 62-69; https://doi.org/10.1111/j.1365-2125.2004. 02183.x

27. E. García-Martín, C. Martínez, B. Tabarés, J. Frías and J. A. Agúndez, Interindividual variability in ibuprofen pharmacokinetics is related to interaction of cytochrome P450 2C8 and 2C9 amino acid polymorphisms, Clin. Pharmacol. Ther. 76 (2004) 119-127; https://doi.org/10.1016/j. clpt.2004.04.006

28. D. Ochoa, R. Prieto-Pérez, M. Román, M. Talegón, A. Rivas, G. Galicia, F. Abad-Santos and T. Cabaleiro, Effect of gender and CYP2C9 and CYP2C8 polymorphisms on the pharmacokinetics of ibuprofen enantiomers, Pharmacogenomics 16 (2015) 939-948; https://doi.org/10.2217/pgs.15.40

29. R. López-Rodríguez, J. Novalbos, S. Gallego-Sandín, M. Román-Martínez, J. Torrado, J. P. Gisbert and F. Abad-Santos, Influence of CYP2C8 and CYP2C9 polymorphisms on pharmacokinetic and pharmacodynamic parameters of racemic and enantiomeric forms of ibuprofen in healthy volunteers, Pharmacol. Res. 58 (2008) 77-84; https://doi.org/10.1016/j.phrs.2008.07.004

30. M. Karazniewicz-Lada, M. Luczak and F. Glowka, Pharmacokinetic studies of enantiomers of ibuprofen and its chiral metabolites in humans with different variants of genes coding CYP2C8 and CYP2C9 isoenzymes, Xenobiotica 39 (2009) 476-485; https://doi.org/10.1080/00498250902862705

31. A. D. Rodrigues and T. H. Rushmore, Cytochrome P450 pharmacogenetics in drug development: in vitro studies and clinical consequences, Curr. Drug Metab. 3 (2002) 289-309; https://doi. org/10.2174/1389200023337522

32. D. H. Solomon, M. E. Husni, P. A. Libby, N. D. Yeomans, A. M. Lincoff, T. F. Lüscher, V. Menon, D. M. Brennan, L. M. Wisniewski, S. E. Nissen and J. S. Borer, The risk of major NSAID toxicity with celecoxib, ibuprofen, or naproxen: A secondary analysis of the PRECISION trial, Am. J. Med. 130 (2017) 1415-1422.e4; https://doi.org/10.1016/j.amjmed.2017.06.028

33. N. Moore and J. M. Scheiman, Gastrointestinal safety and tolerability of oral non-aspirin overthe-counter analgesics, Postgrad. Med. 130 (2018) 188-199; https://doi.org/10.1016/j.amjmed.2017.06.02 